\title{
A COUNTEREXAMPLE CONCERNING THE EXTREMAL INDEX
}

\author{
RICHARD L. SMITH, ${ }^{*}$ University of Surrey
}

\begin{abstract}
The concept of an extremal index, which is a measure of local dependence amongst the exceedances over a high threshold by a stationary sequence, has a natural interpretation as the reciprocal of mean cluster size. We exhibit a counterexample which shows that this interpretation is not necessarily correct.
\end{abstract}

Let $\left\{\xi_{n}, n=0,1,2, \cdots\right\}$ denote a stationary sequence and define $M_{n}=\max \left\{\xi_{1}, \cdots, \xi_{n}\right\}$. Under suitable conditions it is possible to prove results of the form

$$
n P\left\{\xi_{1}>u_{n}\right\} \rightarrow \tau \Leftrightarrow P\left\{M_{n} \leqq u_{n}\right\} \rightarrow e^{-\theta \tau}(0<\tau<\infty)
$$

where $0 \leqq \theta \leqq 1$. The parameter $\theta$ was termed the extremal index by Leadbetter (1983), though the concept had occurred earlier in papers of Newell (1964), Loynes (1965), O'Brien (1974a), (1974b) and Davis (1982). For a general overview of extremes in stationary sequences, see Leadbetter et al. (1983).

It is possible to define an exceedance point process $N_{n}$ on $(0,1]$, such that $N_{n}(s, t]$ is the number of exceedances of the level $u_{n}$ among $\left\{\xi_{r}: n s<r \leqq n t\right\}$. Convergence of $\left\{N_{n}\right\}$ as $n \rightarrow \infty$ is studied by Hsing et al. (1988). One of their main results is that, if a limiting point process exists, then it must be compound Poisson. The atoms of this limiting process correspond to clusters of exceedances. Somewhat parallel results have also been obtained by Alpuim (1987).

A natural interpretation of $\theta$ is that $1 / \theta$ is the mean cluster size in the limiting point process. Hsing et al. were not, however, able to prove this without making additional assumptions. The following example shows that the result is false without such assumptions.

The example is a regenerative sequence of the form

$$
\xi_{n}=\zeta_{j} \text { for } \sum_{0}^{j-1} N_{i} \leqq n<\sum_{0}^{j} N_{i}(j \geqq 1)
$$

where

(i) $\zeta_{j}, j \geqq 1$ are independent with a common distribution function $F$ satisfying $F(1)=0$, $F(x)<1$ for all $x<\infty$,

(ii) For $j>1$, given $N_{1}, \cdots, N_{j-1}, \zeta_{1}, \cdots, \zeta_{j}$ with $m \leqq \zeta_{j}<m+1$, the probability of the event $N_{j}=i$ is $q_{m i}$. Here $\left\{q_{m i}, m \geqq 1, i \geqq 1\right\}$ is a sequence of probabilities with $q_{m i} \geqq 0$, $\Sigma_{i} q_{m i}=1$ for each $m$.

In words, the process remains in state $\zeta_{i}$ for a random number of time epochs determined by the probability distribution $q_{m i}(i \geqq 1)$ with $m=\left[\zeta_{i}\right]$, and then moves to a new state which is independently chosen from $F$.

Let $p_{m}=P\left\{m \leqq \zeta_{j}<m-1\right\}, \mu_{m}=\Sigma_{i} i q_{m i}$ and suppose $\mu=\Sigma p_{m} \mu_{m}<\infty$. Then $\mu$ is the mean recurrence time of the process. The process may be made stationary by a suitable choice of

Received 27 January 1988; revision received 6 May 1988.

Postal address: Department of Mathematics, University of Surrey, Guildford, GU2 5XH, UK.

Supported by Air Force Office of Scientific Research at University of North Carolina; Grant Number F $4962085 \mathrm{C} 0144$. 
distribution of $N_{1}$. It may also be regarded as a function of a Harris chain, and therefore be treated by extreme value arguments of O'Brien (1987) and Rootzén (1988).

Now let us specify $\left\{q_{m i}\right\}$ to be

$$
q_{m i}= \begin{cases}(m-1) / m, & i=1, \\ 1 / m, & i=m+1, \\ 0, & \text { otherwise. }\end{cases}
$$

Then $\mu_{m}=2$ for all $m$, and so $\mu=2$ also. Let $\left\{u_{n}, n \geqq 1\right\}$ be a sequence of thresholds such that, $u_{n} \rightarrow \infty, n P\left\{\zeta_{1}>u_{n}\right\} \rightarrow \tau, 0<\tau<\infty$.

Proposition 1. $\theta=\frac{1}{2}$.

Proof. Immediate from Theorem 3.1 of Rootzén (1988).

Proposition 2. The exceedance point process $N_{n}$ converges to a simple Poisson process of intensity $\frac{1}{2}$, as $n \rightarrow \infty$.

Proof. This follows from Theorem 4.2 of Hsing et al. (1988). Positive recurrent Harris chains are strong mixing (cf. O'Brien (1987)), and hence satisfy the mixing condition $\Delta\left(u_{n}\right)$ used by Hsing et al. For a suitable sequence $\left\{r_{n}\right\}$ satisfying $r_{n} \rightarrow \infty, r_{n} / n \rightarrow 0$, let

$$
\pi n(j)=P\left\{\sum_{i=1}^{r_{n}} \chi_{n, i}=j \mid \sum_{i=1}^{r_{n}} \chi_{n, i}>0\right\}, \quad j=1,2, \cdots
$$

where $\chi_{n, i}$ is 1 if $\xi_{i}>u_{n}, 0$ otherwise. Let $\pi(j)=\lim _{n \rightarrow \infty} \pi_{n}(j)$ for $j=1,2$, . The theorem of Hsing et al. asserts that the point process $N_{n}$ converges to a compound Poisson process with compounding distribution $\pi(\cdot)$. Theorem 3.3 of Rootzén (1988) could also be used to give the same result.

However, under (3) it is easy to see that $\pi(j)=1$ for $j=1,0$ for $j>1$. Hence the limiting process in this case is simple Poisson, with a mean cluster size of 1 . This completes our description of the example.

From a statistical point of view, the most natural way to estimate the extremal index is via the point process of high-level exceedances. Such a procedure was in fact proposed by Smith (1984). The example here reveals a possible fallacy in this procedure, though it may not be possible to do much about it in practice.

\section{Remarks}

1. What is going wrong is the lack of tightness of the sequence $\left\{q_{m i}, i \geqq 1\right\}$ as $m \rightarrow \infty$. In a similar way it is possible to construct an example of the same phenomenon for any $\theta<1$. Hsing et al. show that the extremal index is in general given by $\theta=\lim _{n}\left\{\Sigma_{j} j \pi_{n}(j)\right\}^{-1}$, so what is at issue is whether

$$
\Sigma j \pi_{n}(j) \rightarrow \Sigma j \pi(j) \quad \text { as } \quad n \rightarrow \infty
$$

This is false for the example considered here.

2. It is possible to exhibit other kinds of extremal behaviour by taking other sequences $\left\{q_{m i}\right\}$. For instance, if $\mu_{m} \rightarrow \infty$ we very easily obtain an example of a process with extremal index 0 . Taking this one step further, if $q_{m i} \rightarrow 0$ as $m \rightarrow \infty$ for each $i$ but the distribution $\left\{q_{m i}, i \geqq 1\right\}$ converges under some renormalisation to the distribution of a continuous random variable as $m \rightarrow \infty$ (example: take $q_{m i}=1 / m$ for $\left.i=1,2, \cdots, m\right)$, then the point process $N_{n}$ does not converge but a suitably renormalised sequence converges to a compound Poisson point process with continuous compounding distribution. Such behaviour is admitted in the general theory of Hsing et al., but they do not give any examples. 


\section{Acknowledgement}

This work was carried out during a visit to the Center for Stochastic Processes, University of North Carolina. I am grateful to Ross Leadbetter, Tailen Hsing and Teresa Alpuim for helpful discussions.

\section{References}

AlPUIM, M. T. (1987) High-level exceedances in stationary processes with index. Preprint, University of Lisbon.

Davis, R. A. (1982) Limit laws for the maximum and minimum of stationary sequences. $Z$. Wahrscheinlichkeitsth. 61, 31-42.

Hsing, T., HüsLeR, J. AND LeAdBetTer, M. R. (1988) Limits for exceedance point processes. Prob. Thy. Rel. Fields. To appear.

LEADBETTER, M. R. (1983) Extremes and local dependence in stationary sequences. Z. Wahrscheinlichkeitsth. 65, 291-306.

Leadbetter, M. R., Lindgren, G. ANd Rootzen, H. (1983) Extremes and Related Properties of Random Sequences and Series. Springer-Verlag, New York.

LOYNES, R. M. (1965) Extreme values in uniformly mixing stationary stochastic processes. Ann. Math. Statist. 36, 993-999.

NewEll, G. F. (1964) Asymptotic extremes for $m$-dependent random variables. Ann. Math. Statist. 35, 1322-1325.

O'Brien, G. L. (1974a) Limit theorems for the maximum term of a stationary process. Ann. Prob. 2, 540-545.

O'Brien, G. L. (1974b) The maximum term of uniformly mixing stationary processes. $Z$. Wahrschlichkeitsth. 30, 57-63.

O'Brien, G. L. (1987) Extreme values for stationary and Markov sequences. Ann. Prob. 15, 281-291.

Rootzén, H. (1988) maxima and exceedances of stationary Markov chains. Adv. Appl. Prob. 20, 371-390.

SMITH, R. L. (1984) Threshold methods for samples extremes. In Statistical Extremes and Applications, ed. J. Tiago de Oliveira, Reidel, Dordrecht, 621-638. 